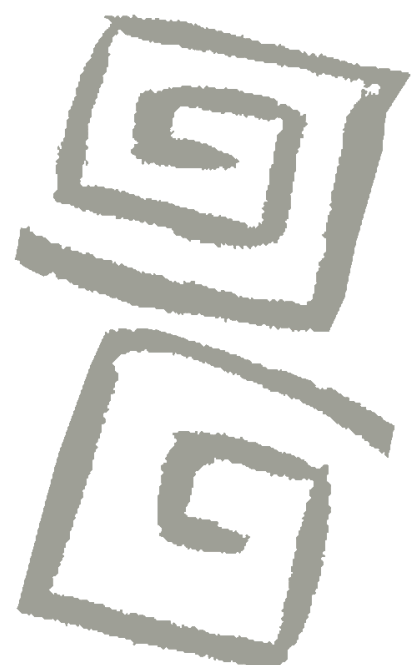

\title{
Modelo integral comunitario para prevenir y abordar problemáticas de salud adolescente
}

\section{A comprehensive community model to prevent and address adolescent health problems}

Ana Clara Camarotti ${ }^{1}$, Gabriela Wald ${ }^{2}$, Alejandro Capriati ${ }^{3}$, Ana Lía Kornblit ${ }^{4}$

${ }^{1}$ Doctora en Ciencias Sociales. Investigadora adjunta, Consejo Nacional de Investigaciones Científicas y Técnicas, con sede en el Instituto de Investigaciones Gino Germani, Universidad de Buenos Aires. Ciudad Autónoma de Buenos Aires, Argentina. $\triangle$ iD

${ }^{2}$ Doctora en Ciencias Sociales. Investigadora adjunta, Consejo Nacional de Investigaciones Científicas y Técnicas, con sede en Instituto de Investigaciones Gino Germani, Universidad de Buenos Aires. Ciudad Autónoma de Buenos Aires, Argentina. $\triangle$ iD

${ }^{3}$ Doctor en Ciencias Sociales. Investigador asistente, Consejo Nacional de Investigaciones Científicas y Técnicas, con sede en Instituto de Investigaciones Gino Germani, Universidad de Buenos Aires. Ciudad Autónoma de Buenos Aires, Argentina. $\triangle$ (iD)

${ }^{4}$ Doctora en Antropología. Investigadora, Instituto de Investigaciones Gino Germani, Universidad de Buenos Aires. Ciudad Autónoma de Buenos Aires, Argentina. $\square$ (iD
RESUMEN En este artículo presentamos una modelización que tiene como meta la construcción de una respuesta integral y comunitaria frente a problemáticas de salud adolescente. Desde un abordaje integral, que incluye acciones de prevención y promoción, asistencia y protección, y que involucra a diferentes sectores y áreas del Estado y de la comunidad, describimos las bases conceptuales del modelo, los ejes y los principios que recuperamos de los debates de la promoción de la salud, la salud colectiva y de las experiencias de trabajo del equipo de investigación en distintas comunidades a lo largo de los últimos 15 años en Argentina. Posteriormente, damos cuenta de los pasos desarrollados para facilitar la realización de intervenciones y retomamos algunos nudos teóricos, políticos y prácticos que se encuentran en su gestación, en una apuesta por vincular la reflexión crítica con el desarrollo de tecnologías sociales. Este modelo da cuenta de la reflexión sobre cómo llevar adelante acciones de promoción de la salud que busquen transformar las realidades particulares, respetando sus singularidades y recuperando lo que las conecta con otras.

PALABRAS CLAVES Prueba de Modelo Conceptual; Promoción de la Salud; Adolescentes; Investigación Participativa Basada en la Comunidad; Argentina.

\begin{abstract}
This article presents a model for building a comprehensive community response to adolescent health problems. The approach is comprehensive in that it includes prevention and promotion, assistance and protection, and involves different sectors and areas of the State and the community. We describe the conceptual framework and principles of the model, developed based on debates regarding health promotion and collective health as well as the work experiences of the research team in different communities in Argentina over the last 15 years. We then present the steps developed to help facilitate interventions and analyze certain theoretical, political and practical issues underlying the management of such a model, in an attempt to link critical reflection with the development of social technologies. This model takes into account reflections regarding how to carry out health promotion actions that seek to transform particular realities, respecting their singularities but at the same time acknowledging their connections to others.
\end{abstract}

KEY WORDS Concept Model Proof; Health Promotion; Adolescents; Community-Based Participatory Research; Argentina. 


\section{INTRODUCCIÓN}

El modelo que presentamos en este artículo está pensado para la promoción de la salud integral de las y los jóvenes y toma como punto de partida el ámbito comunitario. Contiene una síntesis del trabajo de nuestro equipo del área de salud del Instituto de Investigaciones Gino Germani, Universidad de Buenos Aires, tanto en la investigación como en la asistencia técnica de programas de intervención en distintas comunidades a lo largo de los últimos 15 años.

El modelo se inserta en los debates sobre la promoción de la salud, la salud colectiva, y la medicina social latinoamericana, rescatando sus principales aportes y haciendo foco, a su vez, en la dimensión territorial comunitaria. Utilizamos el concepto de modelo porque lo entendemos como un instrumento metodológico cuya utilidad es la interpretación de las prácticas. Los modelos suponen un alto nivel de abstracción y solo deben considerarse como referencias teóricas de situaciones concretas, históricamente determinadas.

El mismo se afianzó en nuestro último proyecto de promoción de la salud integral adolescente en Valles Calchaquíes, titulado Hacia un sistema de acción regional integrado.-Fortalecimiento del trabajo y las acciones de las instituciones locales y las redes juveniles (2014-2018), en el cual acompañamos el trabajo de un grupo de organizaciones sociales y gobiernos locales y contamos con el apoyo de Unicef Argentina. Si bien esa iniciativa tuvo como eje la prevención del abuso sexual contra niños, niñas y adolescentes y el mejoramiento del sistema de respuestas a dicha problemática, el modelo que presentamos puede ser utilizado para trabajar un abanico amplio de temas de salud integral.

Esta modelización tiene como meta la construcción de una respuesta integral y comunitaria frente a problemáticas de salud adolescente. Hablamos de un abordaje integral porque se incluyen acciones de prevención y promoción, asistencia y protección, en las cuales se involucran a diferentes sectores y áreas del Estado y de la comunidad. Enfatizamos la movilización comunitaria porque los procesos de transformación son más duraderos cuando se compromete a las poblaciones $y$, en el caso que nos ocupa, cuando las y los adolescentes se asumen como protagonistas del cambio que quiere llevarse a cabo.

Este modelo es una tecnología de intervención social pensada para ser utilizada por distintos grupos sociales. No es neutra porque subyace un marco teórico e ideológico de participación, de toma de la palabra y de acciones por parte de la comunidad y, progresivamente, de las y los adolescentes y jóvenes. Asimismo, porque entiende la salud como un proceso colectivo, que incluye tanto la salud como la enfermedad y el cuidado; considera la juventud no en términos negativizantes, sino como potencialidad de acción; se trabaja desde una perspectiva de derechos humanos y de género que busca desnaturalizar las miradas heteronormativas. Como veremos a lo largo de este trabajo, todos estos supuestos se explicitan y discuten en los procesos de formación de equipos territoriales de adultos y jóvenes. No desconocemos que no todos los grupos sociales tienen las mismas posibilidades de influir en las agendas locales de implementación, tanto de temas como de modelos de intervención. Sabemos que organismos de cooperación internacional y organizaciones de la sociedad civil con acción globalizada cuentan con estrategias de incidencia basadas en una legitimidad construida internacionalmente. No obstante, sobre la base de distintas experiencias de trabajo sabemos también que las organizaciones sociales locales comprometidas con su comunidad son capaces de igualar esos niveles de legitimidad (confianza ganada a partir del trabajo cotidiano con las comunidades) y convertirse en engranajes claves para llevar adelante este tipo de propuestas.

A continuación, presentamos los ejes conceptuales y los principios del modelo en diálogo con los debates de la promoción de la salud, la salud colectiva y de las experiencias de trabajo realizadas en las comunidades con el propósito de sentar sus bases conceptuales. Para tal fin, hemos sintetizado diez 
principios, organizados en tres ejes conceptuales: a) nada se sostiene en el tiempo sin la movilización comunitaria, la articulación entre instituciones y el compromiso del Estado; b) adolescentes y jóvenes como protagonistas del cambio, con apoyo de adultos que estimulan y cuidan; y c) diagnosticar, planificar, evaluar y sistematizar de manera participativa.

Posteriormente, presentamos los seis pasos que hemos desarrollado para facilitar la realización de proyectos o intervenciones bajo este modelo: 1) diagnóstico participativo y formulación de problemas; 2) organización y formación de las y los participantes jóvenes y adultos; 3 ) comunicación del proyecto a los actores de la comunidad y áreas del Estado vinculadas; 4) trabajo en las escuelas; 5) acciones en la comunidad; 6) articulación entre instituciones y la construcción de una respuesta social.

En este trabajo hacemos foco en los ejes conceptuales y principios del modelo. Analizamos en ellos los "porqué" del tipo de intervención que proponemos. Los pasos del modelo, los cuales presentamos de manera sintética en este trabajo, implican avanzar sobre el "cómo" de las acciones. Estos pasos suponen una dialéctica que, por un lado, da lugar para que lo singular y contingente de cada experiencia pueda tomar lugar. No obstante, reconocen también que muchos problemas se repiten de modo similar en distintas comunidades, ya sea cercanas o geográficamente alejadas. La apuesta es a no encorsetar soluciones, pero a tomar en cuenta los aprendizajes que puedan iluminar zonas o espacios comunes de acción.

\section{Implicancias de la movilización comunitaria para el abordaje de la salud y los derechos en la adolescencia}

Haciendo un poco de historia encontramos como referencia tres modelos claves que se han utilizado para pensar las acciones de prevención en salud en el mundo: el modelo de la historia natural de la enfermedad y los niveles de prevención, el de la nueva promoción de la salud y el de vulnerabilidad y derechos humanos, desarrollado en las últimas décadas por colegas brasileños ${ }^{(1)}$.

El modelo de la historia natural de la enfermedad fue utilizado para analizar cómo contribuía el medio ambiente a las enfermedades, comparando lugares, épocas, estaciones del año, características geográficas, poblacionales, etc. Hacia mediados del siglo XX la discusión se trasladó de Inglaterra a EEUU y Leavell y Clark se constituyeron en sus referentes, demostrando que las enfermedades eran multicausales. Postularon entonces que las medidas curativas no debían escindirse de las preventivas, así como la clínica médica no podía pensarse ajena a la salud pública. En esta línea, desarrollaron la idea de que la prevención debía atravesar todas las instancias en las cuales se pudiera intervenir en salud, y propusieron los niveles de prevención (primaria, secundaria y terciaria).

Hacia la década de 1970, un movimiento potente de ideas vinculado a un nuevo concepto de promoción de la salud intentó renovar las prácticas de salud, desarrollando una política de trabajo entre saberes científicos y técnicos en diálogo con las poblaciones, de modo que estas aumentaran el control sobre todo aquello que pudiera afectar su salud y su cuidado. No obstante el consolidado marco teórico y las reiteradas declaraciones de organismos internacionales realizadas durante las últimas décadas en el marco de conferencias internacionales, el campo de la promoción de la salud continúa enfrentando dificultades para su aplicación. El foco aún individualista y conductista de las acciones que en su nombre se emprenden, el privilegio de las dimensiones biomédicas de las acciones de promoción y educación para la salud que aún impera en las intervenciones, así como las dificultades para el trabajo intersectorial, son sus grandes asuntos pendientes ${ }^{(2,3,4)}$.

Hacia la década de 1990 surge en Brasil un grupo de académicos comprometidos con la intervención, quienes introdujeron conceptos de las ciencias sociales para pensar la acción en salud, dando lugar al enfoque de vulnerabilidad y derechos humanos ${ }^{(5,6)}$. Definen vulnerabilidad como "un conjunto de 
aspectos individuales y colectivos relacionados a la mayor susceptibilidad de individuos y comunidades ante una enfermedad o daño, $y$, de modo inseparable, menor disponibilidad de recursos para su protección"(1). Este enfoque busca comprender tanto las bases sociales como las implicancias éticas y políticas que subyacen a las prácticas de cuidado -o su omisión- de las personas y comunidades. Finalmente, al ser un enfoque de derechos humanos, enfatiza la responsabilidad de los Estados y gobiernos en los procesos de salud. Este modelo afirma que cuantas más vulnerabilidades se observen en un territorio o comunidad, menor será el impacto de las acciones de prevención y promoción de la salud. Esto no quiere decir que no se pueda trabajar en prevención y promoción en estas comunidades, sino que los resultados estarán mediados por los determinantes sociales de los procesos de salud-enfermedad-cuidado.

Uno de sus puntos novedosos es que estos enfoques incluyen en el análisis de dichos procesos los modos de intervenir sobre estos, siendo que las concepciones, saberes, tecnologías y servicios en salud de los cuales se disponga determinarán los procesos de salud-enfermedad-cuidado. De este modo, la intervención deja de pensarse como la solución, pasa a ser considerada parte del problema en estudio y conceptualizan el proceso de salud-enfermedad como un proceso continuo de salud-enfermedad-cuidado. De esta manera, se ocupa de los sentidos e implicancias de las relaciones sociales en la inequidad de posibilidades de enfermar, así como de analizar cuánto los Estados regulan y protegen los derechos humanos.

En línea con este enfoque y las ideas de la promoción de la salud se enmarca la presente modelización. Partimos de una definición amplia e integral de la salud, entendida como un proceso colectivo, influido por aspectos económicos, políticos, psicosociales, culturales y ambientales. El modelo que proponemos busca promover aspectos que mejoren la calidad de vida de las y los adolescentes, así como el abordaje de las problemáticas vinculadas a los contextos sociales en los cuales se desarrollan sus vidas, sin olvidar los aspectos subjetivos que viabilizan sus aspiraciones y el fortalecimiento de sus capacidades.

La modelización toma como eje central de intervención la dimensión comunitaria, entendida como un espacio social en construcción permanente, atravesado en distintos grados por la diversidad, el conflicto y la cooperación. No se parte de una perspectiva romántica de la comunidad, en tanto armónica y homogénea, sino como un espacio atravesado por relaciones de poder entre actores e instituciones que coexisten en un territorio. No obstante, se propone potenciar vínculos positivos entre los grupos sociales con los que se trabaja, sin desestimar la existencia de intereses contrapuestos. Se busca entonces maximizar las potencialidades del trabajo colaborativo, a la vez que minimizar la influencia de los intereses particulares o factores que no colaboran con el desarrollo de la acción colectiva, entendida como la acción que privilegia el bien común.

Antes de adentrarnos en el modelo, queremos llamar la atención sobre los límites de este tipo de intervenciones y de sus alcances. Hay quienes desde una postura crítica afirman que, en tanto no mejoren las condiciones estructurales que determinan los problemas de salud adolescente, no se lograrán modificaciones sustantivas en relación con la calidad de vida de las y los jóvenes, que está en la base de dichos problemas o, en el mejor de los casos, se alcanzará solo un paliativo. Siguiendo a Onetto ${ }^{(7)}$, en los proyectos comunitario-sociales es posible diferenciar una "zona de preocupación" y una "zona de incumbencia". La primera comprende todos los factores que pueden influir, en este caso, sobre la salud integral adolescente, las cuales escapan a nuestro radio de acción como operadores comunitarios, pero que sabemos que tienen una influencia decisiva sobre ella como, por ejemplo, el nivel socioeconómico y cultural de las familias de los y las adolescentes. La zona de incumbencia, en cambio, es la que está directamente en el foco de lo que podemos modificar, por ejemplo, la posibilidad de que los y las jóvenes defiendan su derecho a la educación sexual 
integral. Teniendo en cuenta precisamente la posibilidad de las intervenciones que pueden incentivar cambios, preferimos Ilamarla zona práctica de influencia. Si bien no podemos modificar las condiciones laborales, la calidad educativa, el acceso a la seguridad social, etc., sí es posible generar transformaciones intermedias: reflexión crítica de la comunidad, movilización para conseguir que se amplíen derechos o que se hable sobre temas silenciados, trabajo con instituciones claves (como la escuela, el centro de salud, organismos de gobierno, etc.) para mejorar los sistemas de respuestas a determinada problemática, la generación de vínculos entre adolescentes y adultos que redunden en vínculos de cuidado, entre otras.

Por último, debemos tener en cuenta en el desarrollo de las acciones en salud un enfoque de género y de derechos, incluyendo los sociales, económicos y culturales. Este enfoque es crucial para el reconocimiento y el respeto de la dignidad y la capacidad de acción de las y los adolescentes, su ciudadanía y participación activa, asumiendo que no existe una sola forma de vivir la adolescencia sino múltiples, y que la experiencia de ser joven depende de cuestiones estructurales y culturales, como también de las relaciones sexo genéricas.

\section{DESARROLLO DE LOS EJES CONCEPTUALES Y PRINCIPIOS DEL MODELO}

El Cuadro 1 muestra los tres ejes conceptuales y los diez principios del modelo de intervención para el abordaje de la salud adolescente.

\section{Eje conceptual 1: Nada se sostiene en el tiempo sin la movilización comunitaria, la articulación entre instituciones y el compromiso del Estado}

Durante las últimas décadas, en el campo de la salud, se han propuesto una serie de ideas con algún grado de consenso, tendientes a lograr intervenciones efectivas y sustentables,

\section{Cuadro 1. Ejes conceptuales y principios del modelo de intervención para el abordaje de la salud adolescente.}

\begin{tabular}{|c|c|c|}
\hline Eje conceptual 1 & \multicolumn{2}{|r|}{$\begin{array}{l}\text { Nada se sostiene en el tiempo sin la movilización comunitaria, la articulación entre instituciones y el compromiso } \\
\text { del Estado }\end{array}$} \\
\hline \multirow[t]{4}{*}{ Principios } & 1. & Movilización comunitaria como herramienta de abogacía. \\
\hline & 2. & $\begin{array}{l}\text { Articulación interinstitucional para mejorar el acceso a los servicios de salud, protección, justicia y } \\
\text { programas sociales. }\end{array}$ \\
\hline & 3. & $\begin{array}{l}\text { Búsqueda de apoyo del Estado nacional, provincial y municipal para dar sustentabilidad a las } \\
\text { respuestas locales. }\end{array}$ \\
\hline & 4. & $\begin{array}{l}\text { Ampliación de acciones comunitarias a otras localidades para potenciar las respuestas y reforzar la } \\
\text { sustentabilidad. }\end{array}$ \\
\hline Eje conceptual 2 & \multicolumn{2}{|r|}{ Adolescentes y jóvenes como protagonistas del cambio, con apoyo de adultos que estimulan y cuidan } \\
\hline \multirow[t]{3}{*}{ Principios } & 5. & Adolescentes que tomen la palabra y sean parte de la acción colectiva. \\
\hline & 6. & Escuelas como espacios privilegiados para la promoción de la salud. \\
\hline & 7. & Los cambios en los repertorios culturales sólo son posibles si se propicia la reflexión crítica. \\
\hline Eje conceptual 3 & \multicolumn{2}{|c|}{ Diagnosticar, planificar, evaluar y sistematizar de manera participativa } \\
\hline \multirow[t]{3}{*}{ Principios } & 8. & $\begin{array}{l}\text { Conformación y capacitación de equipos locales para llevar adelante las tareas y unificar criterios de } \\
\text { intervención. }\end{array}$ \\
\hline & 9. & $\begin{array}{l}\text { Monitoreo permanente para cambiar a tiempo lo que no funcione y evaluación para registrar los } \\
\text { cambios ocurridos. }\end{array}$ \\
\hline & 10. & Sistematización para documentar lecciones aprendidas. \\
\hline
\end{tabular}


que hacen hincapié en la participación y empoderamiento de las comunidades en general (sumando adolescentes y jóvenes si se trata de acciones que los/las interpelen), en la intersectorialidad, en la necesidad de acciones multiestratégicas que impliquen saberes y acciones de distintos alcances y disciplinas $y$, en el caso de la adolescencia, se suma la necesidad de contar con entornos amigables para que los y las jóvenes se acerquen ${ }^{(1,8,9,10)}$. El modelo que presentamos está en línea con estas ideas, y enfatiza la trilogía que supone la movilización comunitaria, la centralidad del Estado y el involucramiento de las organizaciones de la sociedad civil. No obstante, esta sinergia necesaria entre actores, instituciones y modos de hacer no es sencilla de establecer, pues no todos parten de los mismos supuestos, ni tienen los mismos intereses, las mismas lógicas de acción, las mismas posibilidades o capacidades para liderar procesos, lo cual redunda muchas veces en desencuentros entre miradas técnicas, políticas, académicas y los saberes del mundo de la vida. Saldar estas dificultades no es sencillo, y requiere de instituciones y líderes comunitarios dispuestos al diálogo, así como de encuentros en los que se puedan consensuar objetivos prioritarios y marcos teórico-metodológicos. Pues es fundamental para el trabajo comunitario poder sintetizar miradas y formas de hacer, las cuales tiendan a construir puentes y no a zanjar brechas cada vez mayores.

Partimos del supuesto de que no hay a priori una institución u organización (o un tipo de institución) que detente la legitimidad para liderar un proceso utilizando el modelo que estamos proponiendo. Si bien un organismo de cooperación internacional de renombre puede ser bien recibido en un comienzo por una comunidad, luego tendrá que mostrar en su hacer que sus credenciales y sus formas de intervenir concuerdan con aquello que se espera de él. En sentido inverso, una organización que se gesta en el seno de la comunidad con referentes que muestran compromiso y entrega en la tarea cotidiana logrará el reconocimiento suficiente como para accionar convocando a otras organizaciones, incluso al mismo Estado. En cada territorio, el poder y la legitimidad se disputan en las acciones cotidianas. Si las organizaciones dan respuestas en el momento en el que se las necesita, si ofrecen una escucha honesta y desinteresada, si se involucran y consiguen resolver necesidades concretas de las personas, todo eso sienta bases que son tanto o más fuertes que el renombre previo para quien esté dispuesto a intervenir.

El derecho de las y los adolescentes a ejercer niveles cada vez mayores de responsabilidad y autonomía en lo que refiere a su vida y a la de sus comunidades no reduce las obligaciones de los Estados de garantizar su protección, lo que incluye garantizar el pleno ejercicio de sus derechos sociales, económicos y culturales ${ }^{(11)}$.

La movilización comunitaria implica trabajar con todos los actores que tengan un compromiso con el cuidado de la salud integral de las y los adolescentes, y con la promoción de sus derechos.

En este caso, además, es preciso recalcar el anclaje territorial de las acciones (instituciones y organizaciones conectadas para potenciar la prevención, la promoción y el cuidado) así como la necesidad de no aplicar recetas (en cada territorio y comunidad las acciones serán singulares, atentas al contexto sociocultural y a los antecedentes en el trabajo comunitario). Una comunidad dispone de bienes económicos, redes afectivas, técnicas o maneras de hacer, recursos, conocimientos, experiencias de trabajo en red, valores morales: a todo ello se debe apelar para la realización de un proyecto que busque encontrar soluciones a problemas comunes $^{(12)}$.

En este tipo de proyectos es necesario trabajar en tres niveles. En el nivel político: acciones para que los gobiernos e instituciones locales apoyen el proyecto que se va a desarrollar y su perspectiva de trabajo. Nivel técnico: acciones de capacitación, desarrollo de materiales, asesoría en temáticas específicas, investigación, monitoreo, evaluación y puesta en red de experiencias de promoción de la salud. Nivel operativo: es el momento 
de pensar: ¿qué tipo de actividades hacer?, ¿Cómo vincular distintos tipos de problemáticas?, ¿cómo establecer vínculos duraderos entre instituciones y entre los actores de las mismas?

\section{Principio 1: Movilización comunitaria como herramienta de abogacía}

Una comunidad movilizada tiene más herramientas para demandar al Estado los derechos incumplidos, así como para cuidar y proteger a sus miembros. La acción colectiva se construye en la intersubjetividad, en los vínculos con otros, los cuales, como hemos dicho, implican muchas veces aprendizajes significativos, pero muchas otras negociaciones complejas para llegar a consensos que estarán en permanente renegociación.

La movilización involucra a adolescentes y jóvenes, tanto adentro como afuera de la escuela, y a adultos o familias que decidan acompañarlos. Con una comunidad movilizada todas las actividades están enmarcadas en un proyecto mayor que se difunde, se prioriza en la agenda local, se instala en las instituciones comprometidas y se comparte con otras comunidades con problemas similares.

\section{Principio 2: Articulación interinstitucional para mejorar el acceso a los servicios de salud, protección, justicia y programas sociales}

Es sabido que cuanto más articuladas estén las instituciones de una comunidad, más protegidos están sus miembros. Pero también sabemos de la dificultad que conlleva lograr la articulación. Para ello es necesario que los actores de las diversas instituciones entiendan la importancia y los beneficios del trabajo en red y se den tiempo para el encuentro y para pensar en los mejores modos de vincularse.

Dado el complejo mundo en el que se desarrolla la vida cotidiana en la actualidad, los recursos o apoyos que enmarcan su desarrollo deben ser producto de articulaciones de un conjunto de instituciones: familia, escuela, servicios de salud, organizaciones comunitarias, clubes y otras organizaciones, todas las cuales pueden resumirse en la expresión adultos que cuidan y estimulan.

Asimismo, es preciso contar con servicios -de salud, pero también de protección y programas sociales- que sean amigables y sepan trabajar con la especificidad que la adolescencia requiere. Servicios en los cuales las y los jóvenes se sientan cuidados y respetados, donde se priorice la confidencialidad y la escucha sin juicio. Para todo ello hace falta que los actores estatales y no estatales modifiquen las percepciones estereotipadas con las que interpelan a las personas jóvenes y logren verlas como agentes de cambio con potencialidad para contribuir en todos los niveles.

\section{Principio 3: Búsqueda de apoyo del Estado nacional, provincial y municipal para dar sustentabilidad a las respuestas locales}

La sustentabilidad de acciones de movilización comunitaria puede lograrse mediante acuerdos con las provincias y municipios de los territorios donde se realizan las intervenciones, de modo que la capacidad que se logró construir quede instalada. Para ello, resulta necesario que el Estado pueda brindar su apoyo a las organizaciones, el cual puede darse de diversas maneras, ya sea financiando algunas actividades, instalando recursos humanos o de otro tipo en lugares en donde no los había y, en algunos casos, si la intervención es muy exitosa, adoptarla como política pública. Es importante el encuentro entre el Estado y las organizaciones comunitarias porque mientras que estas últimas logran una cercanía territorial, el Estado puede garantizar la continuidad y sostenibilidad de las respuestas elaboradas al calor de las comunidades. Si bien la participación comunitaria es uno de los pilares de la sustentabilidad, el otro es el apoyo del Estado, capaz de sostener recursos y otras capacidades en el tiempo. 


\section{Principio 4: Ampliación de las acciones comunitarias a otras localidades para potenciar las respuestas y reforzar la sustentabilidad}

Una intervención local muchas veces se transforma en un acontecimiento puntual, que además puede ocurrir aislado territorialmente, en particular, si se trata de una región alejada de los centros urbanos.

Mucho se ha discutido durante las últimas décadas sobre "lo local" y "lo global". Al mismo tiempo que se ha impuesto a nivel mundial la tendencia a la globalización en los aspectos económicos y sociales, se ha descubierto la potencialidad de los ámbitos locales en los procesos de estructuración de las identidades ciudadanas, teniendo en cuenta que brindan mayores posibilidades de participación y democratización. Entre ambos contextos se están tejiendo nuevas relaciones, no solo en el plano de lo económico, sino fundamentalmente en el plano cultural y humano. Lo que sucede en un barrio o localidad excede habitualmente su ámbito. Existen dinámicas mayores que contienen y que explican aquello que ocurre y queremos modificar. Por eso, es preciso poner en contexto la singularidad del lugar con su entorno más amplio, en el cual suelen repetirse problemáticas similares.

Regionalizar supone salir del aislamiento, trabajar en conjunto para fortalecer los sistemas de respuestas locales, los cuales rara vez implican solo a un barrio o, en áreas semirurales, a una localidad. Regionalizar invita a las comunidades a ampliar la comprensión del fenómeno que desean modificar, a tomar conciencia de que lo que sucede en el territorio en el que se vive también sucede en territorios vecinos. Por eso, uno de los indicadores de los logros de un proyecto comunitario es el acrecentamiento de los vasos comunicantes, aquellos que ligan a la comunidad con otros actores, otras localidades, otras redes.

Además del enriquecimiento mutuo que esta transferencia implique, hay que tener en cuenta que cuantas más localidades abarque una intervención, más chances tendrá de movilizar recursos, de ser escuchada y reconocida, de proponer cambios que logren sustentarse en el tiempo. Una intervención de múltiples barrios o localidades, o incluso regional, tendrá más potencia para generar transformaciones.

\section{Eje conceptual 2: Adolescentes y jóvenes como protagonistas del cambio, con apoyo de adultos que estimulan y cuidan}

Los procesos de cambio en las comunidades son más duraderos si participan adultos y adolescentes del lugar, desde el comienzo del proceso, incluyendo la etapa diagnóstica. ¿Pero cuál es el rol del mundo adulto en la movilización de los y las jóvenes por un objetivo que implique un cambio en la comunidad? Hay que tener en cuenta que la participación adolescente en proyectos comunitarios implica una oportunidad para su propio desarrollo y crecimiento. $Y$ aquí es donde, parafraseando a Donald Winnicott ${ }^{(13)}$, es crucial la presencia adulta: donde haya un adolescente pujando por crecer debe haber un adulto que ofrezca un sostén para su empuje.

Es en lo que denominamos zona práctica de influencia en la que nos planteamos trabajar con jóvenes a nivel comunitario y grupal estimulando solidaridades y también, en términos de sus subjetividades, la posibilidad de soñarse a sí mismos en otros mundos y de pensarse a futuro, rompiendo las ataduras que los mantienen limitados a rutinas empobrecedoras. Las relaciones que una persona pueda hacer entre su pasado, su presente y su futuro vinculan su estado actual con sus posibles identidades en un futuro más alejado.

Desarrollar estas posibilidades depende de los escenarios que ofrecen las condiciones de vida de las y los jóvenes, así como de las expectativas y caminos alternativos que puedan imaginar y concretar en el marco de dichas condiciones. Lo que permite el desarrollo de adolescentes es, en términos del educador ruso Lev Vygotsky ${ }^{(14)}$, la posibilidad de trazar trayectorias de identificación a través de la interacción, al participar en las 
actividades de su mundo. Para que esto suceda, alguien -los "adultos que cuidan"(15), los "apoyos" o "soportes"(16,17) o "andamiajes"(14), como queramos Ilamarlos-, deben haber establecido relaciones entre posibles futuros próximos y futuros más lejanos.

Uno de los puntos centrales para lograr la movilización comunitaria es la integración de jóvenes y adultos, lo cual implica la mutua valoración de intereses y opiniones. Sería deseable que ambos pudieran verse como otros con quienes vale la pena el intercambio, de esta manera se construirán vínculos significativos que impactarán de manera positiva tanto en unos como en otros, generando aprendizajes duraderos. Para ello hace falta una escucha atenta, comprometida y que tienda a la integración de los adolescentes en las instituciones y actividades pensadas para ellos.

La posibilidad de concretar acciones colectivas impacta de manera positiva en las posiciones de las y los adolescentes en el mundo. Les muestra que son capaces, que pueden hacer cosas por ellos y por sus pares, que tienen posibilidades de construir de manera colectiva.

Los adultos deben propiciar la reflexión sobre cuál es su lugar y en qué medida el modo en que cumplen su rol habilita o no la participación genuina de los adolescentes, da lugar a su voz y, fundamentalmente, a sus prácticas, aun cuando estas les puedan generar rechazo o incomodidad. Andrés Fuentes, integrante del colectivo de investigación "Ver qué onda", marca una diferencia entre la incomodidad como producto de un asombro moral, y la incomodidad como una sorpresa que nos hace dudar de los supuestos que ordenan nuestra existencia. El asombro moral es un impacto por una situación que nos resulta extraña, que no podemos encajar en nuestro modo preconcebido de entender el mundo. En cambio, la sorpresa, si bien manifiesta una incomodidad, también pone en duda la naturalidad de los principios con los cuales codificamos los hechos que cambian $^{(18)}$. Las dudas e incertidumbres de los adultos frente al mundo cambiante en el que nos toca vivir pueden llevar, por un lado, a adoptar posturas rígidas y censurantes frente a conductas adolescentes que les parecen inadmisibles, pero también pueden llevar a adoptar una postura de tolerancia extrema frente a las conductas contestatarias, basada en un difuso sentimiento de culpabilidad por el mundo que les hemos dejado. En ambos casos no estaremos cumpliendo con lo que las y los adolescentes necesitan: que sostengamos de un modo firme y no rígido el lugar de las normas, lo que permite marcar límites y saber con claridad cuáles son las "reglas de juego", qué es lo que la convivencia social requiere aceptar y hasta dónde puede Ilegar la confrontación para que resulte innovadora y no destructiva.

\section{Principio 5: Adolescentes que tomen la palabra y sean parte de la acción colectiva}

Las intervenciones comunitarias deben apelar al fortalecimiento de estas y otras capacidades. El desarrollo de algunos recursos instrumentales les puede permitir tomar responsabilidad de algunos aspectos de la acción colectiva, logrando autonomía y conexión con el mundo adulto. El trabajo en equipo de adolescentes que se comprometan con el desarrollo de la propia comunidad es el motor de cualquier cambio en cuestiones que los afecten. En primer lugar, porque podrán constituirse en referentes de sus pares en temáticas vinculadas con su bienestar. Luego, porque dicho trabajo brinda la posibilidad de desarrollar empatía en la interacción con otros diversos. Finalmente, porque abre puertas para imaginar y brindar respuestas solidarias en el territorio que se habita.

Si bien en una primera instancia los adultos pueden asumir un protagonismo que ayude a adolescentes jóvenes a organizarse, es preciso transferir estas capacidades de manera gradual al grupo movilizado, en un proceso de autonomía progresiva de su parte.

\section{Principio 6: Escuelas como espacios privilegiados para la promoción de la salud}

A pesar de los cambios que ha sufrido la sociedad en los últimos 25 años en Argentina, 
la escuela sigue siendo la principal institución de referencia para la mayor parte de adolescentes y jóvenes. Además de que permanecen en ella un tiempo considerable, es un espacio de encuentro entre ellos y con el mundo adulto. Es principalmente en la escuela donde muchas y muchos adolescentes establecen vínculos significativos con adultos a quienes toman como referentes, o que los ayudan a atravesar situaciones problemáticas.

Por otra parte, es preciso comprender el valor pedagógico que tiene toda experiencia de promoción de la salud y del cuidado, razón por la cual la escuela y las y los docentes se convierten en espacios y actores claves para llevarlas adelante.

Las escuelas que trabajen en promoción de la salud con adolescentes es importante que logren construir espacios democráticos e integradores. No hace falta convocar al alumno más aplicado y con mejor actitud ante el trabajo escolar. Sería deseable que participaran de la movilización aquellos que deseen hacerlo, de modo de propiciar espacios en los cuales puedan integrarse quienes habitualmente son señalados, identificados como conflictivos y problemáticos. Los espacios que se construyan deben estar abiertos a todas las personas que deseen ser parte, valorando y respetando a cada una en sus capacidades, posibilidades y diferencias.

Finalmente, convocar para el trabajo a padres y madres de la comunidad interesados/as es otro elemento que potencia la movilización de las instituciones escolares.

\section{Principio 7: Los cambios en los repertorios culturales solo son posibles si se propicia la reflexión crítica}

En las intervenciones comunitarias de promoción de la salud se busca propiciar cambios en las prácticas sociales, lo que en gran medida también transforma sus repertorios culturales. La modificación de dichos repertorios y de las prácticas asociadas a ellos no se logra a través de la persuasión, sino que requiere la participación activa de los sujetos en el proceso de cambio. Es preciso reflexionar sobre las raíces culturales, interaccionales y sociales que enmarcan las prácticas que queremos modificar. Limitar este proceso a lo que se ha denominado "modelo informativo" no ha dado buenos resultados. Un ejemplo de esto es proponer el uso del preservativo dando información acerca de cómo se usa, etc., sin contemplar las situaciones de poder que cruzan las relaciones de pareja y que pueden obstaculizar que las mujeres logren acordar con los varones el uso. O el trabajo con "expertos", quienes muchas veces tienen una mirada sobre lo que se debe hacer que no es compatible con las concepciones de las comunidades. Esto ocurre en gran medida cuando adultos no preparados desde lo pedagógico-relacional, sino solo desde su saber específico, les hablan a las y los jóvenes.

Si bien contar con información precisa es necesario, es aún más importante alentar las capacidades de las comunidades mediante la reflexión crítica, para que puedan actuar sobre las circunstancias que las afectan y ayudarlas a generar procesos de cuidado.

En conjunto con la reflexión crítica en las comunidades, es importante identificar códigos y escenas institucionales que no son propicias para abordar el problema que se quiere trabajar: por ejemplo, el médico que da por supuesta la orientación sexual hegemónica de un adolescente que consulta, o el docente que da clases expositivas sin promover la búsqueda activa del aprendizaje. Asimismo, es importante identificar repertorios positivos para la promoción y el cuidado de las comunidades frente a la problemática seleccionada para el trabajo: ¿qué es lo que deberían incorporar las instituciones?, ¿qué es lo que sí pueden ofrecer?

\section{Eje conceptual 3: Diagnosticar, planificar, evaluar y sistematizar de manera participativa}

La base de toda acción de movilización comunitaria es la participación de dicha comunidad en todas las fases del proceso, desde la etapa diagnóstica hasta la planificación y ejecución de acciones. Entendemos la participación como un proceso en el cual se involucra a 
los actores de una comunidad no solo para conocer su opinión, sino también para que formen parte de los procesos de toma de decisiones. Grupos de jóvenes, organizaciones de la comunidad, profesionales y no profesionales, dependencias del Estado vinculadas a adolescencia y juventud, servicios de salud, escuelas, investigadores y centros académicos, todos deben ser convocados para esta etapa.

Así como es importante diagnosticar y planificar con los aportes de los distintos actores de una comunidad, también lo es que puedan ser parte de los procesos de monitoreo, evaluación y sistematización ${ }^{(19,20)}$. No obstante, no todos pueden hacer todas las tareas. El modelo que proponemos implica la conformación de equipos de trabajo locales que reúnan actores que estén trabajando temáticas de adolescencia y juventud, de modo que la mayor cantidad de organizaciones e instituciones estén representadas en dicho equipo o articulen con él.

Las intervenciones comunitarias no parten de un grado cero de organización, sino que, partiendo de aquello que existe, identifican de modo colectivo aquello que puede mejorarse, que debe cambiarse, y aquello que hay que crear para mejorar lo existente ${ }^{(12)}$.

\section{Principio 8: Conformación y capacitación de equipos locales para llevar adelante las tareas y unificar criterios de intervención}

Los procesos de movilización comunitaria que implican a la población adolescente precisan de un equipo de trabajo que lleve a cabo las tareas de manera sistemática y con compromiso cotidiano. Para ello, es necesario que del encuentro entre actores locales que tuvo lugar para la realización del diagnóstico y la planificación participativa se defina qué actores y/o instituciones van a llevar adelante el proceso, en articulación con los demás actores de la comunidad.

Es fundamental que haya un equipo de trabajo que coordine las acciones a realizar, el cual puede ser un equipo ya existente de una de las organizaciones locales o que se cree para llevar a cabo el proyecto que se desea implementar.
Una vez conformados los equipos, es preciso realizar encuentros de formación con tres objetivos principales: a) discutir y acordar un marco de conceptos y modos de hacer compartido; b) establecer al interior del equipo los papeles, funciones y responsabilidades de cada persona o grupo; c) definir el rumbo de la acción colectiva.

\section{Principio 9: Monitoreo permanente para cambiar a tiempo lo que no funcione $y$ evaluación para registrar los cambios ocurridos}

El monitoreo es el seguimiento de actividades, procesos y resultados que se realiza durante un proyecto, el cual permite revisar de manera sistemática lo que está ocurriendo y, por tanto, lo que sería deseable que continuara o que se modificara. Es un insumo indispensable tanto para la gestión administrativa como estratégica de una intervención.

La evaluación es la emisión de un juicio de valor acerca de una política o un programa/proyecto. Puede ser entendida como:

- Una actividad programada de reflexión sobre la acción que pueda compararse en distintas etapas del proyecto (antes-después; antes-durante-después). Se lleva a cabo mediante procedimientos sistemáticos de recolección, análisis e interpretación de información y a través de comparaciones respecto de parámetros definidos. Sirve para orientar la acción ${ }^{(19)}$.

- Un proceso reflexivo que se apoya en la formulación de preguntas precisas sobre uno o varios aspectos relativos al diseño, ejecución o finalización de una intervención ${ }^{(21)}$.

Tanto el monitoreo como la evaluación son fundamentales para orientar la acción, para la toma de decisiones a mediano y largo plazo, a la vez que son útiles para el aprendizaje de quienes forman parte de un programa o proyecto. 


\section{Principio 10: Sistematización de la experiencia para documentar lecciones aprendidas}

La sistematización de experiencias implica una interpretación crítica a partir de un ordenamiento y una reconstrucción de elementos objetivos y subjetivos que han intervenido en un proceso para comprenderlo, interpretarlo y así aprender de la propia práctica. Se reconstruye la información sobre los actores y factores que han intervenido, sobre cómo se han vinculado entre sí y, en la medida de lo posible, se analiza por qué motivos lo han hecho de esa manera. La clave es avanzar en una interpretación crítica del proceso de movilización comunitaria realizado que permita extraer aprendizajes que tengan utilidad para el futuro.

Si bien es importante reconstruir históricamente la experiencia vivida, la sistematización implica entender por qué se pudo pasar de una etapa a la otra en un proyecto, y qué es lo que explica las continuidades y las discontinuidades que pudieron haber ocurrido.

Es habitual que en los proyectos aparezca la necesidad de cambio y, a la vez, surjan resistencias. En el proceso de sistematización es importante avanzar hacia una comprensión de la dialéctica entre cambios y resistencias. Haber vivido la experiencia constituye el punto de partida de la sistematización, pero también el punto de quiebre entre sistematización e investigación: mientras se investiga lo desconocido, se sistematiza lo conocido (lo vivido).

\section{PASO A PASO: DEL DIAGNÓSTICO A LA SISTEMATIZACIÓN}

Los pasos que presentamos a continuación son una forma de implementar los principios que animan el trabajo comunitario en salud con jóvenes (Figura 1), no es la única, pero sí

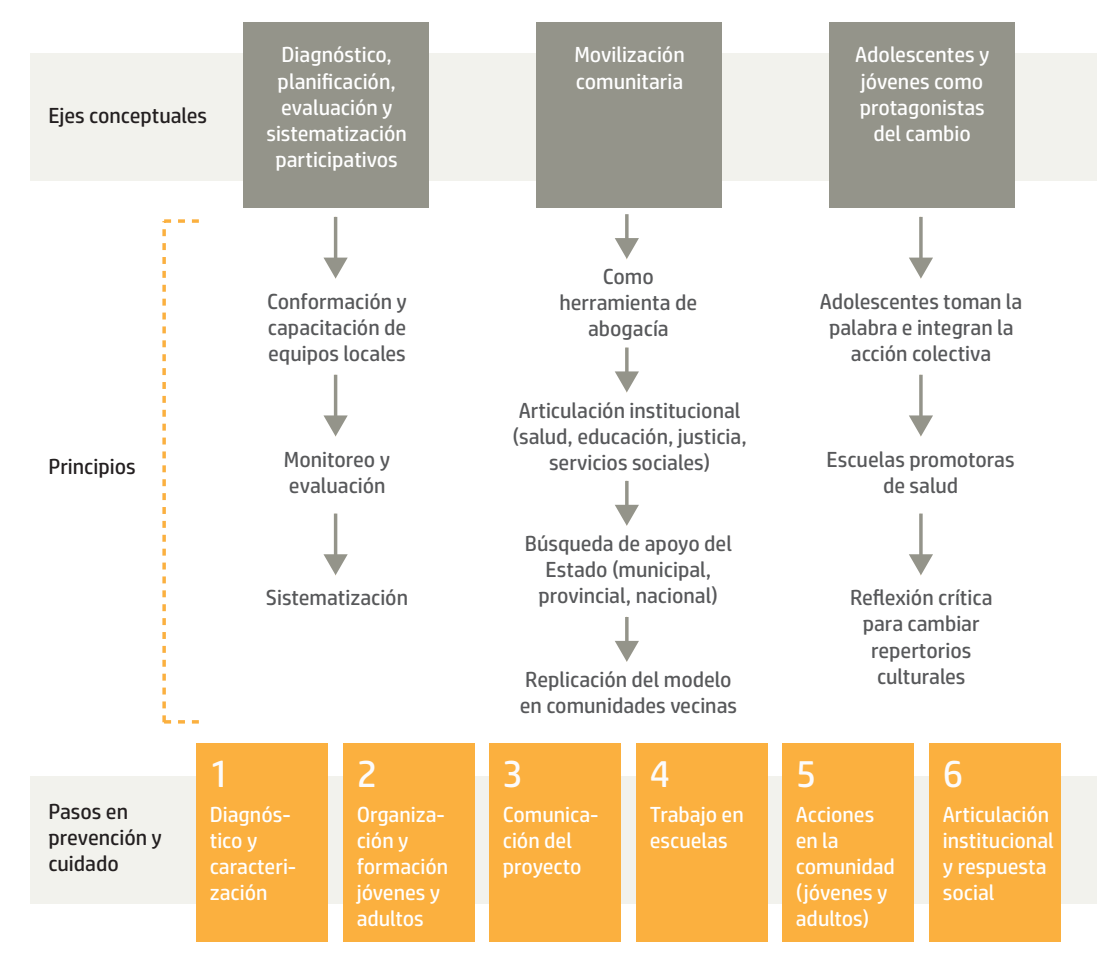

Figura 1. Pasos del diagnóstico a la sistematización: modelo de intervención para el abordaje de la salud adolescente.

Fuente: Elaboración propia. 
es un modo que ha bridando resultados positivos en tanto: a) facilita la realización de acciones integrales, b) alienta la participación genuina de adolescentes y jóvenes y c) promueve el trabajo intersectorial a partir del vínculo entre las instituciones y los referentes comunitarios de un territorio. Cada nueva iniciativa reflexionará de modo colectivo cuáles son los pasos pertinentes y necesarios en función del escenario que afrontan, de los recursos que dispongan y de los grupos, equipos e instituciones que estén dando forma a una nueva experiencia comunitaria.

\section{Paso 1: Diagnóstico participativo y caracterización de cuestiones de salud integral adolescente}

El diagnóstico participativo implica el acercamiento entre organizaciones, el Estado y otros grupos de personas de una comunidad para priorizar los principales problemas vinculados a cuestiones de salud y derechos en la adolescencia, pensar modos de encararlos y coordinar la acción de manera conjunta. En este proceso, es importante reunir la información disponible y la evidencia científica con los conocimientos teóricos, empíricos y vivenciales de todos los involucrados en el tema. A partir del diagnóstico, se priorizarán los temas a trabajar y se hará una selección para comenzar a abordar algunos de ellos.

El diagnóstico y la priorización presentan dos grandes desafíos. Por un lado, que las instituciones y actores relevantes de una comunidad se sientan convocados por la iniciativa y aseguren su participación, aun cuando sus orientaciones políticas o partidarias sean contrastantes. Esto implica convocar y comprometer a todo aquel que trabaje cuestiones vinculadas a la salud integral adolescente. En segundo lugar, hace falta contar con la presencia y la participación de adolescentes y jóvenes de la comunidad, lo cual constituye un desafío doble: que se acerquen y que hagan oír su voz. Para ello es preciso, por un lado, generar una convocatoria amplia en distintos ámbitos donde los y las adolescentes circulan (la escuela, el club, el centro cultural, el comedor, organizaciones comunitarias, etc.) y, por el otro, que cuando los adolescentes se acerquen, el mundo adulto pueda ofrecerles espacios en donde se combine la reflexión con la distensión.

En este paso se busca lograr un consenso acerca de la selección del o los aspectos de la problemática a trabajar: formulación clara de objetivos y roles de las instituciones y grupos participantes, formulación de estrategias para alcanzar la articulación institucional y de un cronograma tentativo de acción.

La priorización de los temas a trabajar se realiza a partir de un proceso colaborativo y participativo, que supone poner un asunto en el orden del día de la atención pública de una comunidad, darle la importancia adecuada, subrayar su centralidad y su significatividad. La priorización se basa, por un lado, en la evidencia científica existente con relación a las problemáticas de la región -en este caso de salud adolescente- $y$, por otro lado, del debate de expertos, técnicos, académicos, profesionales de la salud y de la educación, informantes claves y grupos de adolescentes previamente organizados como, por ejemplo, los centros de estudiantes.

La información que se debate sobre las distintas temáticas permite trascender los acontecimientos que puedan preocupar en un territorio e integrarlos a un contexto social, económico y político, y a un marco interpretativo que lo vincula a otros acontecimientos o fenómenos ${ }^{(22)}$.

No obstante, la decisión final de cuál será el o los tema a trabajar implica una combinación de factores y no es lineal, pues en ella inciden tanto aspectos vinculados a la magnitud y la frecuencia con la que ocurre un determinado problema, la centralidad que el problema tiene para algunos miembros de una comunidad, como los diferenciales de legitimidad que distintos actores de un proceso de priorización participativo puedan tener para instalar un tema de trabajo como, por ejemplo, quién financia la iniciativa. 


\section{Paso 2: Organización y formación de las y los participantes jóvenes y adultos}

Este paso supone la capacitación de las y los adolescentes que quieran ser parte del proyecto, así como de los representantes de las organizaciones e instituciones comunitarias que participarán activamente. Se busca consensuar el marco general de trabajo en la promoción y el cuidado de la salud, los contenidos conceptuales específicos del o los temas priorizados y las estrategias posibles para abordarlo en la comunidad de manera integral.

Se trata de encuentros entre equipos técnicos y no técnicos, grupos de jóvenes, trabajadores del Estado, referentes comunitarios, investigadores, docentes y profesionales para acordar una mirada, establecer funciones y responsabilidades y generar un plan de acción colectivo.

En los encuentros se puede trabajar con adolescentes y adultos en sesiones compartidas o por separado. El desafío de la tarea conjunta es doble: 1) que las y los adolescentes no reproduzcan en sus opiniones las posiciones del mundo adulto, 2) que el grupo adulto no censure la expresión de los grupos de jóvenes.

\section{Paso 3: La comunicación del proyecto a la comunidad y a las nuevas instituciones vinculadas}

La comunicación del proyecto incluye dos aspectos: la instalación del tema que se trabajará en la comunidad y la sensibilización para lograr el compromiso por parte de las organizaciones de la comunidad e instituciones estatales en sus distintos niveles (municipales, provinciales, nacionales). Si bien la etapa diagnóstica implicó una primera convocatoria a organizaciones vinculadas con los y las adolescentes y jóvenes, en este paso se intentará sumar a nuevas instituciones que no hayan sido convocadas para la etapa diagnóstica o que, por distintos motivos, no se hubieran sumado a participar. A partir del nivel de involucramiento, las organizaciones que formaron parte de la etapa diagnóstica van conformando una mesa chica de trabajo que es la que colaborará para sumar nuevas instituciones en este tercer paso.

La comunicación del proyecto atraviesa todos los pasos del modelo propuesto y la idea es que sea exponencial, que implique ir sumando nuevos actores, y que tenga como objetivo final un alcance lo más masivo posible en el territorio, comprometiendo cada vez a más personas y a más instituciones. La instalación de un tema en la comunidad es un paso central tanto para la prevención, como para mejorar la respuesta social, y para demandarle al Estado que haga cumplir los derechos de las y los adolescentes.

\section{Paso 4: El trabajo en las escuelas}

Dado que la escuela es una institución fundamental en la formación de niños, niñas y adolescentes, en cuanto a su rol como ciudadanos comprometidos en pos de una sociedad más justa y equitativa, es importante trabajar con docentes y estudiantes en el modelo de promoción de la salud con enfoque comunitario que proponemos. Para que los proyectos en promoción de la salud sean efectivos, se trabaja no solo con el modelo informativo -aquel que presenta datos e información sobre los daños a la salud-, sino con el modelo de empoderamiento. Este modelo alienta la capacidad de las personas para actuar sobre sus circunstancias a través de técnicas de aprendizaje participativo, reconociendo a los sujetos como protagonistas en el debate y promoviendo la acción colectiva.

No desconocemos que la escuela hoy en Argentina es una institución muy demandada, que interviene en distintas problemáticas sociales que exceden lo estrictamente pedagógico. No obstante, la escuela posee un valor preventivo que ninguna otra institución puede igualar, pues interconecta lo institucional, lo familiar y lo social.

En este modelo, la participación de los y las docentes, así como de la escuela, es voluntaria. Se busca identificar personas aliadas dentro de las instituciones educativas, 
capacitarlas, brindarles herramientas para que puedan ir instalando temas y modelos de trabajo en promoción de la salud en sus instituciones. Asimismo, se busca revisar los supuestos que subyacen a las prácticas sobre las que se quiere trabajar, desarmar miradas estereotipadas, interrogar modelos jerárquicos verticalistas, ortodoxos y encontrar los intersticios para introducir miradas y concepciones más democráticas que acepten las diferencias entre los grupos humanos, tanto en la escuela como en otros ámbitos e instituciones de la comunidad.

El trabajo en las escuelas puede iniciarse mediante acciones emprendidas por adultos, pero progresivamente debe incluir el protagonismo de las y los jóvenes, quienes pasan de la categoría de objetos evaluados a la de sujetos con capacidades y potencial de acción. El modelo reconoce que el saber se encuentra compartido entre docentes y estudiantes y, por ende, ambos son indispensables para construir las prácticas de cuidado.

El objetivo de este paso es lograr escuelas comprometidas, que articulen educación con salud y que estén interesadas en desarrollar actividades para promover la sensibilización sobre temas específicos de salud adolescente.

\section{Paso 5: Las acciones en la comunidad}

Una comunidad movilizada tiene más herramientas para demandar al Estado los derechos incumplidos, así como para cuidar y proteger a sus miembros. La movilización involucra a adolescentes y jóvenes, tanto adentro como afuera de la escuela, pero también a adultos y/o familias que decidan sumarse, o acompañar el proceso de sensibilización que se está llevando a cabo en el territorio.

El objetivo de este paso es el involucramiento en la temática y en las acciones propuestas de todos los actores que tengan un compromiso con el cuidado de la salud integral de las y los adolescentes y con la promoción de sus derechos.

\section{Paso 6: La articulación entre instituciones y la construcción de una respuesta social}

La articulación es una herramienta fundamental que se da a distintos niveles: entre instituciones estatales como escuela, servicio de salud, protección o desarrollo social; entre organizaciones de la sociedad civil e instituciones estatales; entre actividades como promoción, prevención, asistencia y protección; entre jóvenes y adultos; entre disciplinas; etc. Comprender que la acción de cada equipo, servicio o institución forma parte de un engranaje mayor es uno de los principales logros del trabajo comunitario en salud, junto a la habilitación de espacios genuinos de participación juvenil. Para ello es necesario que los actores de las diversas instituciones entiendan la importancia y los beneficios del trabajo en red y se den tiempo para el encuentro y para pensar en los mejores modos de vincularse.

Asimismo, es preciso contar con servicios -de salud, pero también de protección y programas sociales- que sean amigables y sepan trabajar con la especificidad que la adolescencia requiere. A partir de una escucha institucional atenta, el reto es articular las necesidades con los recursos existentes en la comunidad, acompañar a las y los adolescentes para que tomen decisiones informadas, orientarlos en la búsqueda de recursos y realizar el seguimiento de situaciones críticas en las cuales se identifican vulneración de derechos.

Por ello es fundamental lograr respuestas integrales frente a situaciones críticas y diseño de estrategias para lograr acuerdos provinciales que colaboren con el fortalecimiento de la respuesta local existente.

\section{A MODO DE CONCLUSIÓN}

El modelo presentado busca promover la salud integral de los y las adolescentes, así como el abordaje de las problemáticas vinculadas a los contextos sociales en los cuales 
sus vidas se desarrollan, sin olvidar los aspectos subjetivos que permitan viabilizar sus aspiraciones y el fortalecimiento de sus capacidades.

Ahora bien, podemos afirmar que a nivel global se ha logrado avanzar con discursos críticos y propositivos sobre lo que "hay que hacer" para promover la salud integral, en general, y entre los y las jóvenes, en particular. La lógica de este trabajo no es plantear soluciones encorsetadas ni políticas sin sujetos $^{(23)}$ sino colaborar críticamente con la teorización a partir de las prácticas y las experiencias.

Este modelo es fruto de una construcción colectiva que lleva años de reflexión a la luz de la acción. Hemos sido parte de un abanico amplio de programas y proyectos de intervención en distintas provincias y regiones del país, y en cada uno de ellos fuimos conversando sobre los modos de hacer con quienes los llevaban adelante junto a nosotros. Recuperamos aquí el fruto del intercambio con actores de comunidades diversas, así como de reflexiones sobre la práctica que hemos puesto en discusión con aportes teóricos de las ciencias sociales aplicadas a la salud. Desde comienzos de la década de 2000, hemos trabajado teorizando sobre la práctica y practicando sobre la teoría, constituyendo este modelo en un nuevo paso en la reflexión no solo de los cómo sino también de los porqué, para qué, los con quién y para quién $^{(23)}$ de cada acción de promoción de la salud con el fin de transformar realidades de comunidades particulares, en lo que tienen de únicas y singulares, pero también en lo que las conecta con otras.

Como hemos afirmado al inicio de este trabajo, las intervenciones que se pueden realizar a la luz de este modelo tienen sus limitaciones. Pueden transformar algunas aristas de la vida de las personas y, para otras, es necesario un cambio macroestructrural. El concepto de zona práctica de influencia nos acerca a lo que sí podemos lograr: la reflexión crítica de una comunidad; su movilización en pos de abogar por los derechos que les corresponden; el involucramiento de instituciones claves como la escuela, la justicia, organismos gubernamentales, etc., para interrogar concepciones culturales naturalizadas e introducir esquemas de acción tendientes a la equidad y la no violencia; la mejora de las respuestas estatales y no estatales a problemáticas concretas; y la creación de lazos significativos entre adolescentes y adultos que redunden en vínculos de cuidado.

El modelo que aquí presentamos no está exento de las dimensiones de la política y especialmente de lo político, retomando la distinción de Chantal Mouffe ${ }^{(24)}$. Los antagonismos y conflictos de las sociedades humanas son algo más que el telón de fondo de esta tecnología social, la cual buscar revertir relaciones de vulnerabilidad y promover la salud de actores subalternos a partir del reconocimiento de sus voces y su participación. Lo político se expresa en tensiones cotidianas que emergen en la efectiva implementación de las acciones, y si bien en los principios y pasos están contempladas, dependen en última instancia de las singulares circunstancias de cada escenario sociohistórico, limitando o potenciando el trabajo comunitario.

Las intervenciones sociales, por lo tanto, no son neutras: el modelo propuesto está asentado en un enfoque de derechos que buscar identificar y alterar las desiguales distribuciones de poder entre géneros y generaciones; la cooperación intersectorial está atravesada por conflictos de intereses en los cuales interactúan actores con recursos y legitimidades heterogéneas, desde la definición de prioridades hasta la evaluación del proceso; los saberes locales existentes en la comunidad, especialmente, el conocimiento acumulado de técnicos, profesionales y referentes sociales, son tan valiosos para iniciativas de este tipo como socialmente desjerarquizados en el diseño convencional de políticas.

Estos nudos críticos forman parte no solo de la agenda de trabajo formal explicitada en el modelo, sino que también aparecen en situaciones concretas de implementación. Una de esas tensiones refiere a las relaciones de poder que estructuran las relaciones entre adolescentes y jóvenes con el mundo adulto y las instituciones. El modelo propuesto 
cambia el foco de la mirada, impugna el punto de vista adultocéntrico para dar lugar y habilitar las diversidades e intereses de adolescentes y jóvenes.

El reconocimiento de que niñas, niños y adolescentes son personas con derechos al igual que los adultos y el desplazamiento de la mirada tutelar remiten a procesos complejos, con marchas y contramarchas a nivel institucional ${ }^{(25)}$. Una de las principales tareas del modelo propuesto es retirar las etiquetas que pesan sobre las y los adolescentes, tanto las que niegan su capacidad de acción como las nuevas que promueven el mandato de hacerse a sí mismos como si fuera simplemente una cuestión de actitud y voluntad, desconociendo el peso de las constricciones sociales y la desigualdad en la distribución de recursos y oportunidades. El reto es cómo alentar el derecho de las y los adolescentes a ejercer niveles cada vez mayores de responsabilidad y autonomía, sin reducir las obligaciones de los Estados ni promover la hiper responsabilización de los sujetos.

Estos nudos teóricos, políticos y prácticos están en la gestación de este modelo. La apuesta por construirlo radica en la necesidad de vincular la reflexión crítica con el desarrollo de tecnologías sociales, para que dicha reflexión contribuya con la construcción de espacios cada vez más democratizantes y justos.

\section{AGRADECIMIENTOS}

Esta investigación ha sido financiada por Unicef Argentina, a través de los siguientes proyectos: "Salud, género y adolescencia en Valles Calchaquíes. Identificación de prioridades y plan de acción (2015-2016)" y "Hacia un sistema de acción regional integrado. Fortalecimiento del trabajo y las acciones de las instituciones locales y las redes juveniles, Valles Calchaquíes (2016-2018)". Nuestro agradecimiento a quienes fueron los protagonistas en este proceso comunitario regional, los integrantes de la "Red Aquí y Ahora a Tu Lado" de Santa María (Catamarca); la comunidad indígena de Amaicha del Valle (Tucumán) y los gobiernos municipales de San Carlos y Cafayate (Salta).

\section{REFERENCIAS BIBLIOGRÁFICAS}

1. Ayres JR, Paiva V, Franca Jr IF. Conceitos e práticas de prevenção: da história natural da doença ao quadro da vulnerabilidade e direitos humanos. En: Paiva V, Ayres JRCM, Buchalla CM, (orgs.). Vulnerabilidade e direitos humanos: prevenção e promoção de saúde. Curitiba: Juruá Editora; 2012. p. 71-94.

2. Polland B. Health promotion in Canada: perspectives and future prospects. Revista Brasileira em Promoção da Saúde. 2007;20(1):3-11.

3. Wald G. Promoción de la salud a través del arte: estudio de caso de un taller de fotografía en "Ciudad Oculta", la villa No 15 de la Ciudad de Buenos Aires. Salud Colectiva. 2009;5(3):345-362.
4. Kornblit AL. La promoción de la salud entre los jóvenes. Acta Psiquiátrica y Psicológica de América Latina. 2010;56(3):217-225.

5. Ayres JRCM, França Jr I, Calazans G), Saletti Fo HC. El concepto de vulnerabilidad y las prácticas de salud. En: Czeresnia D, Freitas CM, (eds.). Promoción de la salud: conceptos, reflexiones, tendencias. Buenos Aires: Lugar Editorial; 2006. p. 135-162.

6. Ayres JRCM. Para comprender el sentido práctico de las acciones de salud: contribuciones de la Hermenéutica Filosófica. Salud Colectiva. 2008;4(2):159-172.

7. Onetto F. Criterios de intervención en las problemáticas de convivencia escolar. Monografías Virtuales: Ciudadanía, Democracia y Valores en Sociedades Plurales [Internet]. 2003;(2) [citado 2 jul 2018]. Disponible en: https://tinyurl.com/y8k7gtsv.

8. World Health Organization. Global accelerated action for the health of adolescents (AA-HA!): guidance to support country implementation [Internet]. Geneva: World Health Organization; 2017 [citado 2 jul 2018]. Disponible en: https:// tinyurl.com/y72y9x6z.

9. World Health Organization. Promoting health in the SDGs; report on the 9th Global conference for health promotion, Shanghai, China, 21-24 November 2016: all for health, health for all [Internet]. Geneva: World Health Organization; 2017 [citado 2 jul 2018]. Disponible en: https:// tinyurl.com/yayxvtx4. 
10. World Health Organization. Estrategia mundial para la salud de la mujer, el niño y el adolescente (2016-2030) [Internet]. Geneva: World Health Organization; 2017 [citado 2 jul 2018]. Disponible en: https://tinyurl.com/y7l79ota.

11. Argentina, Fondo de las Naciones Unidas para la Infancia. Para cada adolescente una oportunidad [Internet]. Buenos Aires: Unicef Argentina; 2017 [citado 2 jul 2018]. Disponible en: https:// tinyurl.com/ycenuhgr.

12. Camarotti A, Kornblit AL. Abordaje integral comunitario de los consumos problemáticos de drogas: construyendo un modelo. Salud Colectiva. 2015;11(2):211-221.

13. Winnicott D. Los procesos de maduración y el ambiente facilitador: estudios para una teoría del desarrollo emocional. Barcelona: Editorial Paidós; 1994.

14. Vygotski LS. Obras Escogidas. Tomo I. Madrid: Aprendizaje Visor, Ministerio de Educación y Ciencia; 1991.

15. Marina JA. Aprender a vivir. Barcelona: Ariel; 2004.

16. Martuccelli D. Cambio de rumbo: la sociedad a escala del individuo. Santiago de Chile: LOM Ediciones; 2007.

17. Martucelli D, De Singly F. Las sociologías del individuo. Santiago de Chile: LOM Ediciones; 2012.

18. Huergo D, Martínez M. Adolescentes y alcohol: tomar hasta caer. Revista Anfibia [Internet]. 2017 [citado 10 ene 2018]. Disponible en: https:// tinyurl.com/y8xdup7o.
19. Nirenberg O. Evaluación y participación: orientaciones conceptuales para una mejora de la gestión. En: Chiara M, Di Virgilio M, Arriagada I, (orgs.). Gestión de la política social: conceptos y herramientas. Buenos Aires: Prometeo; 2009. p. 267-294.

20. Kornblit AL, Camarotti AC, Capriati A, Di Leo PF, Wald G. Abordaje comunitario de los consumos de drogas: una propuesta para sistematizar experiencias [Internet]. Buenos Aires: Teseo; 2016 [citado 2 feb 2018]. Disponible en: https://tinyurl. com/yb8evqwh.

21. Di Virgilio M, Solano R. Monitoreo y evaluación de políticas, programas y proyectos sociales [Internet]. Buenos Aires: Fundación CIPPEC; 2012 [citado 2 feb 2018]. Disponible en: https:// tinyurl.com/y76t2aun.

22. Lang G, Lang KE. Watergate: an exploration of the agenda-building process. En: Wilhoit G, De Bock H, (eds.). Mass communication review yearbook 2. Beverly Hills: Sage; 1981. p. 447-468.

23. Spinelli $H$. Las dimensiones del campo de la salud en Argentina. Salud Colectiva. 2010;6(3):275-293.

24. Mouffe C. En torno a lo político. Buenos Aires: Fondo de Cultura Económica; 2011.

25. Schuch P. Uma lei moderna x uma cultura tradicional: notas sobre reformulação do campo de atenção à infância e juventude no Brasil. Revista Brasileira de História \& Ciências Sociais. 2010;2(4):73-84. 